RASĀYAN J. Chem.

Vol. 14 | No. 3 |1462-1468| July - September | 2021 ISSN: 0974-1496 | e-ISSN: 0976-0083 | CODEN: RJCABP http://www.rasayanjournal.com http://www.rasayanjournal.co.in

\title{
MOLECULARLY IMPRINTED POLYMERIC MICROSPHERES FOR ELECTROCHEMICAL SENSING OF CHOLESTEROL
}

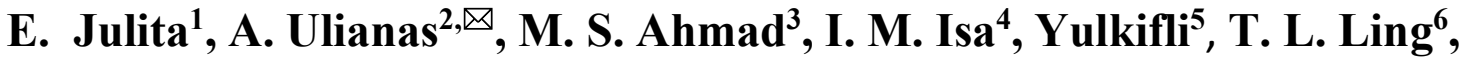 \\ Y. Yolanda ${ }^{7}$, Nurlely ${ }^{8}$ and M. Rezayi ${ }^{9}$ \\ ${ }^{1,2}$ Department of Chemistry, Faculty of Mathematics and Science, Universitas Negeri Padang, \\ Sumatera Barat 25131, Indonesia \\ ${ }^{3,4}$ Department of Chemistry, Faculty of Science and Mathematics, Universiti Pendidikan Sultan \\ Idris, Tanjong Malim 35900, Perak, Malaysia \\ ${ }^{5}$ Department of Physics, Faculty of Mathematics and Science, Universitas Negeri Padang, \\ Sumatera, Indonesia \\ ${ }^{6}$ Southeast Asia Disaster Prevention Research Initiative (SEADPRI-UKM), Institute for \\ Environment and Development (LESTARI), Universiti Kebangsaan Malaysia, 43600 UKM \\ Bangi, Selangor, Malaysia. \\ ${ }^{7}$ Department of Chemistry, University of Ulsan, Ulsan 44610, South Korea \\ ${ }^{8}$ Department of Physic, Faculty of Mathematics and Science, Universitas Indonesia, \\ Jakarta, Indonesia \\ ${ }^{9}$ Department of Medical Biotechnology and Nanotechnology, Faculty of Medicine, Mashhad \\ University of Medical Sciences, Mashhad, Iran \\ Corresponding Author: alizar_chem@yahoo.co.id
}

\begin{abstract}
An electrochemical cholesterol sensor based on emulsion photo-polymerized methacrylic acid molecularly imprinted polymers (MIPs) microspheres modified screen-printed electrode (SPE) has been developed. Electrochemical quantitation of cholesterol concentration was performed by means Cyclic Voltammetry (CV) and Square Wave Voltammetry (SWV) technique by using redox couple of potassium ferricyanide $\left[\mathrm{K}_{3} \mathrm{Fe}(\mathrm{CN})_{6}\right]$. A decrement in the electrochemical CV and SWV signal indicated that the cholesterol molecules were bound to the microspheres' cavities of the biomimetic MIPs sensor, and formed an electron transfer barrier to the redox species from electron transfer at the electrode surface. Under optimal conditions, the electrochemical MIPs microsensor could detect cholesterol concentration in the linear response range of $0.5 \mathrm{mg} \mathrm{L}^{-1}$ to $50.0 \mathrm{mg} \mathrm{L}^{-1}$ with a limit of detection (LOD) at $0.1 \mathrm{mg} \mathrm{L} \mathrm{L}^{-1}$. The MIPs microspheres-modified carbon SPE electrode providing good operational stability to cholesterol sensing of up to 11 days long.
\end{abstract}

Keywords: Molecularly imprinted Polymeric Microsphere, Cholesterol Electrochemical Sensor, Screen-printed Electrode.

RASĀYAN J. Chem., Vol. 14, No.3, 2021

\section{INTRODUCTION}

The main cause of death in both developed and developing countries is coronary artery disease. Some studies have shown an increased risk of cardiovascular disease including atherosclerosis is strongly associated with high blood cholesterol levels (hypercholesterolemia). ${ }^{1}$ Hypercholesterolemia can lead to human health problems such as coronary heart disease, stroke, calcification of blood vessels, etc. Therefore, the determination of cholesterol levels in foods and blood is becoming increasingly important for clinical analysis or diagnosis as well as determining health risk. ${ }^{1}$

Conventional methods for cholesterol measurement involved the use of laboratory analytical instrumental approaches, such as mass spectrometry or liquid and gas chromatography. Although they are highly sensitive and provide the most accurate result, however, they require extensive sample pre-treatment and costly equipment. Fluorometric and colorimetric enzymatic assays, on the other hand, although limits of 
RASĀYAN J. Chem.

Vol. 14 | No. 3 |1462-1468| July - September | 2021

detection (LOD) are usually low but involve the use of the enzymes is costly. ${ }^{2}$ Therefore, simpler and lowcost cholesterol determination methods are greatly demanded, and still give accurate results for assessing the risk of heart disease.

Molecularly imprinted polymers (MIPs) are synthetic materials mimicking molecular recognition elements by antibodies, enzymes, or other biological receptors. ${ }^{3}$ With this, MIPs-based sensors will supersede traditional enzymatic biosensors and immunosensors, which requires the use of animals and a complex and expensive enzyme purification process. MIPs are most selective for the particular print molecule used to prepare them ${ }^{4}$, and that MIPs shows excellent selectivity towards the template molecule as the cavities formed on the MIPs are based on the size, shape and chemical complementary of the imprint molecule. ${ }^{5}$ Thus, MIPs shows high promise as a robust artificial receptor material for sensing applications. ${ }^{6}$

MIPs sensing technique has been appeared as one of the most promising techniques in the field of sensors due to their simplicity, low cost, reliability, availability of a wide selection of templates and functional monomers. The recent development of non-enzymatic MIPs sensor based on metal, metal alloys as well as certain metal oxides modified screen-printed carbon electrodes for hydroquinone determination in cosmetic samples has been reported by Mulyasuryan ${ }^{7}$ with exceptional sensitivity and selectivity performance. Another study conducted by Futura ${ }^{6}$ employing MIPs microspheres demonstrated highly sensitive and specific electrochemical detection of $17 \beta$-Estradiol, which was prepared by coating the micro MIPs on the multi-walled carbon nanotube/gold nanoparticle-modified electrode through a simple photo-polymerization technique. Additionally, in situ electrochemical formations of MIPs materials on the electrode surface purely based on MIPs suspension with high sensitivity have also been reported in the literature. ${ }^{8}$

In this study, a non-enzymatic MIPs approach was used to develop an electrochemical cholesterol sensor. Molecularly imprinted polymeric microspheres based on methacrylic acid (MAA) monomer immobilized on the screen-printed carbon paste electrode (SPE) has been fabricated as a new electrochemical sensor for rapid detection of cholesterol. The hydrophobic MIPs microspheres specific towards cholesterol detection were synthesized via a facile photo-polymerization process in emulsion form. The concentration of cholesterol could be electrochemically determined with the MIPs microspheres-based sensor via adsorption of cholesterol on the immobilized MIPs microspheres through hydrogen bonding and was monitored by cyclic voltammetry method by using potassium ferricyanide $\left[\mathrm{K}_{3} \mathrm{Fe}(\mathrm{CN})_{6}\right]$ as the electrolyte solution.

\section{Chemicals and Instruments}

\section{EXPERIMENTAL}

An ultraviolet transmitter unit emitting UV light at the wavelength of $350 \mathrm{~nm}$ was utilized in the photopolymerization reaction of molecularly imprinted polymeric microspheres. The ultrasonic cleaner was used to sonicate the MIP microspheres mixture. The centrifuge was used to isolate the as-synthesized microMIPs. The Perkin Elmer type spectrophotometer is used to record the Fourier infrared (FTIR) spectrum. Potentiostat (Gamry Instruments) was employed to measure the electrochemical voltammetry response of the imprinted cholesterol sensor on the screen-printed carbon paste electrode (SPE), which acted as the working electrode. In addition, the reference and auxiliary electrodes are used $\mathrm{Ag} / \mathrm{AgCl}$ and Glassy carbon, respectively.

All chemicals used in this research were of analytical grade and were used as received without any further purification. Cholesterol powder was produced by Sigma Life Science. 2-2-dimethoxy-2phenylacetophenone (DMPP) photo-initiator, methacrylic acid (MAA) monomer, ethylene glycol dimethacrylate (EGDMA) crosslinker, sodium dodecyl sulfate (SDS) surfactant, Aqua DM, potassium ferricyanide $\left[\mathrm{K}_{3} \mathrm{Fe}(\mathrm{CN})_{6}\right]$ and acetonitrile $\left(\mathrm{C}_{2} \mathrm{H}_{3} \mathrm{~N}\right)$ solvent were obtained from Aldrich Chemical Company.

\section{Synthesis of Methacrylic Acid-based MIPs Microspheres}

The MIPs microspheres were synthesized using the photo-polymerization technique according to the method reported by Futra $^{6}$ with slight modifications. A mixture containing appropriate amounts of cholesterol, MAA functional monomer, EGDMA crosslinker, DMPP photo-initiator, SDS surfactant and distilled water was sonicated for $10 \mathrm{~min}$ at room temperature $\left(25^{\circ} \mathrm{C}\right)$. The white emulsion formed was then 
RASĀYAN J. Chem.

Vol. 14 | No. 3 |1462-1468| July - September | 2021

polymerized by exposure to UV light under a continuous nitrogen gas stream for another $10 \mathrm{~min}$. The resulting MIPs microspheres obtained were collected via centrifugation at high speed $(13000 \mathrm{rpm})$ for 30 min and washed carefully with deionized water followed by air-dried at ambient temperature. The template was then removed from the MIPs microspheres by washing the polymer with acetonitrile (micro-MIPs B). Non-imprinted polymeric microspheres were also prepared using the same procedure, but without template removal step (micro-MIPs A).

Fabrication of MIPs Microspheres-based Biomimetic Electrochemical Cholesterol Sensor

Figure-1 shows the electrochemical design of the Cholesterol Sensor. MIPs microspheres at $15 \mathrm{mg}$ were added into $1 \mathrm{~mL}$ of deionized water and sonicated at $25^{\circ} \mathrm{C}$ for $10 \mathrm{~min} .5 \mu \mathrm{L}$ of the mixture was later dropcoated on the SPE electrode and dried overnight at room temperature. Absorption of analyte on the MIPs microspheres-modified SPE (micro-MIPs-SPE) was done by incubating the micro-MIPs-SPE in $100.0 \mathrm{mg}$ $\mathrm{L}^{-1}$ cholesterol in $66 \%$ ethanol solution for $30 \mathrm{~min}$. The cholesterol sensor was rinsed with deionized water before electrochemical signal measurement using electrochemical (Cyclic Voltammetry/CV and Square Wave Voltammetry/SWV) techniques within the potential range of $-1.0-1.0$ versus $\mathrm{Ag} / \mathrm{AgCl}$ reference electrode in $10 \mathrm{mM} \mathrm{K}{ }_{3} \mathrm{Fe}(\mathrm{CN})_{6}$ electrolyte solution. ${ }^{9,10}$ Cyclic voltammetry can be used in the characterization of conductive oxides ${ }^{11}$ and as an electrochemical sensor response. The blank control was prepared by exposing the imprinted sensor to $66 \%$ ethanol solution without target cholesterol before the electrochemical measurement was performed.

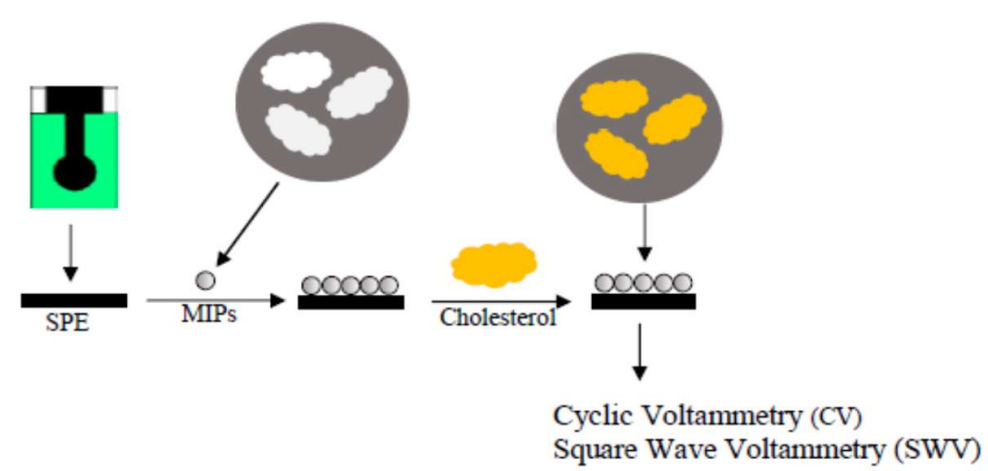

Fig.-1: The Design Mechanism of Electrochemical Cholesterol Sensor based on Methacrylic Microspheres-modified SPE

Optimizing Imprinted Sensor Electrochemical Response for Molecular Recognition of Cholesterol The optimization process allows for achieving optimal setting of parameters for molecular recognition of target cholesterol, which involved template loading effect, $\mathrm{pH}$ effect, response time, dynamic linear concentration of analyte, long-term stability test and sensor repeatability. All the sensor optimization experiments were performed in triplicate.

The amount of template incorporated in the synthesis of micro-MIPs was optimized by changing the cholesterol (template) mass loading between $48.1 \mathrm{mg}$ and $88.1 \mathrm{mg}$ in the micro-MIPs precursor. The respective micro-MIPs obtained were mixed with $1 \mathrm{~mL}$ of deionized water and immediately sonicated at room temperature for $10 \mathrm{~min} .5 \mu \mathrm{L}$ of the respective micro-MIPs' suspensions were then drop-coated on the separate SPE electrodes and dried overnight at $25^{\circ} \mathrm{C}$ before electrochemical SWV response of the MIP sensor was taken with potentiostat for detection of $100.0 \mathrm{mg} \mathrm{L}^{-1}$ cholesterol.

For $\mathrm{pH}$ effect study, a series of cholesterol solutions in $66 \%$ ethanol at $100.0 \mathrm{mg} \mathrm{L}^{-1}$ was prepared at $\mathrm{pH} 6.0$ to $\mathrm{pH} 8.0$ by using $0.1 \mathrm{M} \mathrm{NaOH}$ and $\mathrm{HCl}$ solutions. The imprinted sensors were later dipped into those cholesterol solutions at various pHs, and the SWV response was recorded after $30 \mathrm{~min}$ of reaction time.

Response time of the electrochemical MIPs microsensor was determined by immersing the micro-MIPsmodified electrodes in $100.0 \mathrm{mg} \mathrm{L}^{-1}$ cholesterol solution for $0.5 \mathrm{~min}$ to $30.0 \mathrm{~min}$ at $\mathrm{pH} 7.0$, and the electrochemical response of each SPE was then directly measured with square wave voltammetry method. 
RASĀYAN J. Chem.

Vol. 14 | No. 3 |1462-1468| July - September | 2021

The linear response range of the imprinted microsensor was assessed by using a series of cholesterol solutions at a different concentration between $0.5 \mathrm{mg} \mathrm{L}^{-1}$ and $100.0 \mathrm{mg} \mathrm{L}^{-1}$ at $\mathrm{pH}$ 7.0. Absorption of analyte cholesterol on the MIPs microspheres-immobilized SPE was carried by soaking different micro-MIPs-SPEs into cholesterol solutions at the various concentration for $30 \mathrm{~min}$, followed by washing with deionized water before electrochemical SWV measurement.

A long-term stability test of the molecularly imprinted microsensor was conducted by producing as many as 50 micro-MIPs-based sensors and kept at ambient conditions in a petri dish until further usage. Three MIP sensors were taken for electrochemical measurement to detect $100.0 \mathrm{mg} \mathrm{L}^{-1}$ cholesterol at different days for an experimental duration of 45 days.

\section{RESULTS AND DISCUSSION \\ FTIR Analysis of MIP Microspheres based on Emulsion Photopolymerized}

FTIR analysis was conducted to determine the functional groups in cholesterol, micro-MIPs B with the template being removed with acetonitrile and non-imprinted polymeric microspheres (micro-MIPs A) without template being removed. The FTIR spectra demonstrated in Figure- $2 b$ and Figure- $2 c$ show specific bands correspond to the characteristic absorption bands of the respective cholesterol and methacrylic acid monomer. Upon removal of the template using acetonitrile, the disappearance of the aromatic absorption band associated with the tetracyclic ring of cholesterol at the wavenumber range of $900-690 \mathrm{~cm}^{-1}$ was noted. Based on the comparison of characteristic FTIR bands of functional groups in cholesterol (Figure2a), micro-MIPs A and micro-MIPs B, the non-imprinted micro-MIPs A exhibited prominent out-of-plane bending peaks that appeared in the wavenumber range of $900-690 \mathrm{~cm}^{-1}$, which can be assigned to the carbon ring of cholesterol. The absence of aromatic out-of-plane bending C-H bonds in micro-MIPs B indicates the imprinted sensor materials have been successfully formed as the high-affinity synthetic receptors capable of capturing the target cholesterol.

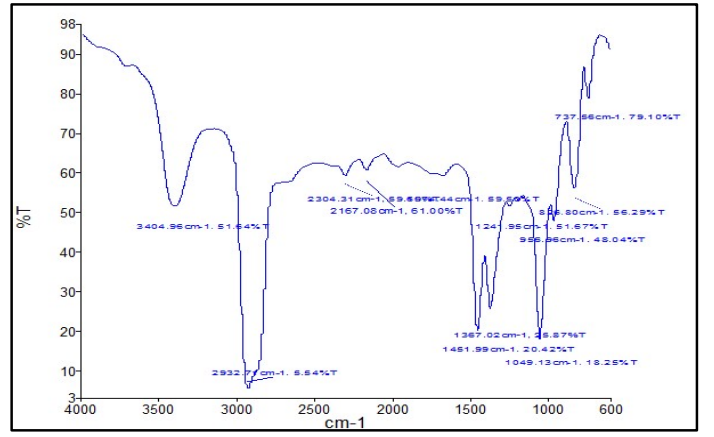

(a)

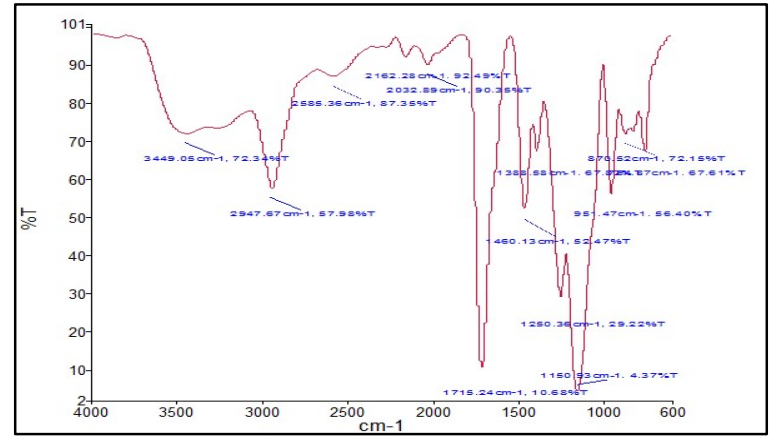

(b)

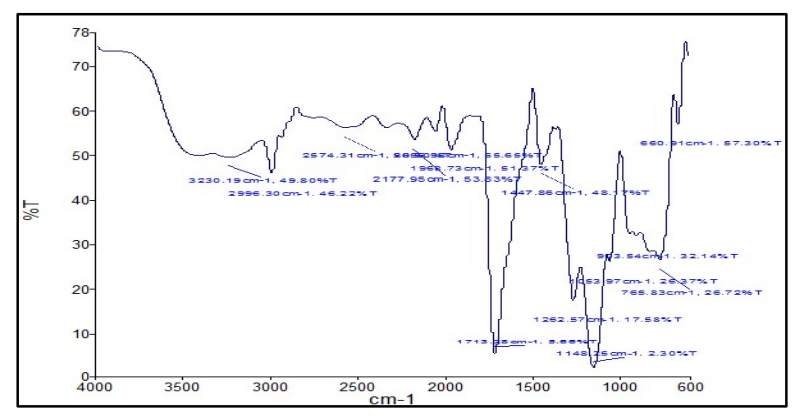

(c)

Fig.-2: FTIR Spectra of Cholesterol (a), Non-imprinted Polymer i.e. microMIPs A without template being removed (b) and MIPs microspheres with the template being removed (micro-MIPs B) (c) with acetonitrile solvent. 
RASĀYAN J. Chem.

Vol. 14 | No. 3 |1462-1468| July - September | 2021

\section{The Electrochemical Response of the MIPs Microspheres-based Cholesterol Sensor}

As explained by Wang ${ }^{13-14}$, the voltammetry technique is an electroanalytical method used in analytical chemistry including examining electroactive chemical compounds, investigating the adsorption process on the electrode surface and studying the electron transfer pathway at the electrode-electrolyte interface, whereby reduction and oxidation reactions occur at the working electrode surface can be transformed into a measurable electrical current. Figure-3 illustrates the cyclic voltammetry response of the imprinted microsensor based on emulsion photo-polymerized methacrylic acid micro-MIPs in $10 \mathrm{mM} \mathrm{K}_{3} \mathrm{Fe}(\mathrm{CN})_{6} \cdot{ }^{10}$ The highest peak current signal was observed for bare SPE ascribed to the redox reaction of $\mathrm{K}_{3} \mathrm{Fe}(\mathrm{CN})_{6}$ electroactive component was taking place at the SPE surface. The unmodified SPE allowed direct electron transfer of the redox species on the electrode surface, hence yielding a significant redox peak current response. However, the redox peak current declined slightly as SPE electrode was modified with MIPs microspheres specific recognition element for cholesterol. The non-conducting characteristic of the microMIPs has somehow restricted electron transfer at the micro-MIPs-SPE electrode surface. The redox peak current response perceived at this stage was attributed to the electron transfer reaction that was occurred at the specific cavities of the MIPs synthetic micro-polymers for their target analyte. When the micro-MIPs' cavities were bound to the cholesterol (analyte), a noticeable decrement in the redox peak current signal was assigned to the inclusion of cholesterol in the micro-MIPs' cavities, which created closed cavities, and further restrained the electron transfer at the electrode surface. ${ }^{15}$

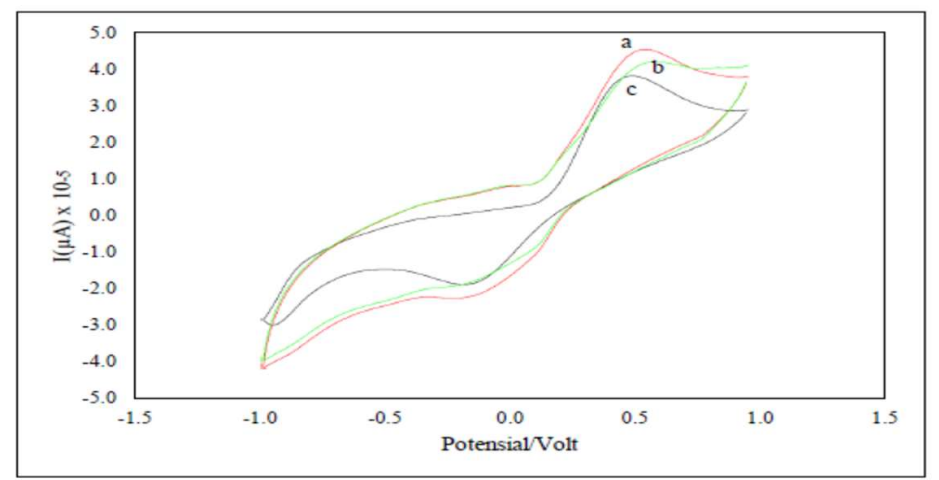

Fig.-3: Cyclic Voltammograms of bare SPE and micro-MIPs-SPE Electrode before and after exposure to $100.0 \mathrm{mg}$ $\mathrm{L}^{-1}$ Cholesterol. The lectrochemical measurement was conducted in the presence of $10 \mathrm{mM} \mathrm{K}_{3} \mathrm{Fe}(\mathrm{CN})_{6}$ with a scan rate of $100 \mathrm{mV} \mathrm{s}^{-1}$.

\section{Template Loading Effect on the Synthesis of MIP Microsphere Synthetic Receptors}

The electrochemical SWV response of the MIPs sensor decreased as increasing the mass value of the template from $48.1 \mathrm{mg}$ to $78.1 \mathrm{mg}$. By increasing the template loading, it promoted an increasing number of imprinted sites for absorption of cholesterol molecules (analyte), and that it formed an electron blocking layer to obstruct electron transfer of the redox couples at the electrode surface, thereby reducing the electrochemical imprinted sensor response. No significant change in the SWV response of the MIPs-based sensor as increasing the template loading to $88.1 \mathrm{mg}$ when the MIPs microsorbents have entirely saturated with specific binding sites for target cholesterol. Therefore, cholesterol loading at $78.1 \mathrm{mg}$ was selected as optimum template loading in the synthesis of high-affinity MIPs microsphere synthetic receptors.

\section{Effect of pH on the Cholesterol Absorption on the Imprinted Microsensor}

The absorption capacity of the immobilized micro-MIPs for cholesterol (analyte) was found to be lower in acidic conditions due to the competitive interaction of proton and analyte for cavities of the micro-MIPs to form intra-cavity proton bonding, and this competitive replacement had rendered a higher electron transfer rate of $\mathrm{K}_{3} \mathrm{Fe}(\mathrm{CN})_{6}$ redox species at the micro-MIPs-SPE, as such higher SWV response of the imprinted microsensor was obtained in the acidic reaction medium. The loading capacity of target cholesterol was maximum at neutral $\mathrm{pH}$ due to the absence of excess competing proton for binding with imprinted cavities ${ }^{16}$ 
and the redox couples were hindered from electron transfer to the significant level at the already occupied micro-MIPs' cavities. Thus, a lower electrochemical response was observed at $\mathrm{pH}$ 7.0. While at basic medium, the SWV response of the micro-MIPs sensor slightly enhanced as the MIPs microspheres may be hydrolyzed to form a hydroxide precipitate and considerably affected the structure and function of the MIPs, therefore interfered with the absorption capacity of the micro-MIPs electrode. ${ }^{17}$

\section{Response Time of the Micro-MIPs Sensor towards Voltammetric Detection of Cholesterol}

The effect of the incubation time of the imprinted microsensor in cholesterol solution at $\mathrm{pH} 7.0$ was investigated to determine the response time of the electrochemical cholesterol sensor. The response of the MIPs microspheres-based electrochemical sensor declined progressively with increasing sensor incubation time from 3-15 min. This suggests the greater amount of cholesterol molecules were absorbed on the MIPs microspheres via hydrogen binding of between oxygen moiety of carbonyl functional group of micro-MIPs and hydroxyl functional group of cholesterol as the incubation time increased ${ }^{6}$. The electron transfer barrier becomes greater as the micro-MIPs-analyte interaction rate increased with increasing the MIPs electrode incubation time, and that gradually reduced the electron transfer rate at the micro-MIPs-SPE surface. A saturation state was attained when the sensor incubation time was prolonged to $30 \mathrm{~min}$, whereby the binding sites of micro-MIPs with high affinity to cholesterol were fully occupied, and the electron transfer rate of redox species achieving a constant rate at this stage. The observed kinetic curve according to Ji report ${ }^{8}$ is typical of most of the rebinding process and expressing cholesterol fast dynamic adsorption to the sensor.

\section{Linear Calibration Range of the micro-MIPs-modified SPE Electrode for Cholesterol}

The MIPs microspheres are generally capable to bind to a certain amount of molecular analyte with the available imprinted sites on the imprinted sensor itself. As can be seen in Fig.-4, the molecularly imprinted microsensor exhibited a linear decreasing SWV response upon exposure to increasing cholesterol (analyte) concentration from $0.5-50.0 \mathrm{mg} \mathrm{L}^{-1}$. The linear electrochemical sensor response with increasing analyte concentration has gradually increased the micro-MIPs-analyte reaction rate, thus declining the electron transfer rate at the MIPs electrode surface in a proportional manner as a result of the inclusion of target analyte in the MIPs microspheres' cavities, thereby forming an obstacle to the electron transfer at the electrode surface. No significant change in the SWV response of the electrochemical MIPs sensor when further increased the cholesterol concentration to $10.0 \mathrm{mg} \mathrm{L}^{-1}$, which can be attributed to the limited imprinted sites on the immobilized micro-MIPs that prevented further absorption with larger amount of target molecules. Limit of detection (LOD) of this sensing system was obtained at $0.1 \mathrm{mg} \mathrm{L}-1$ cholesterol by using the solid-state electrode based on emulsion photopolymerized methacrylic acid MIPs microspheres.

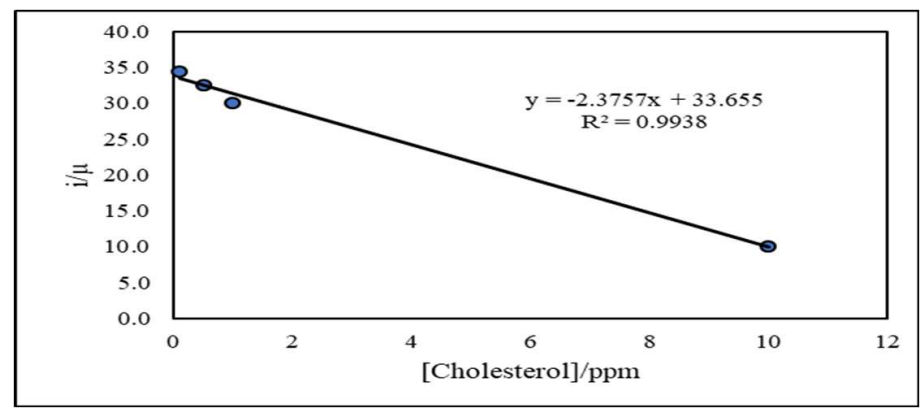

Fig.-4: Linear Response of Cholesterol Sensor

The stability of the MIPs sensor is an important factor for producing a reproducible response towards the detection of cholesterol. ${ }^{8}$ The MIPs sensor was noticed to be able to remain its original electrochemical response for 10 days of storage period at room temperature with no significant change in the SWV signal. About $80 \%$ of the initial imprinted sensor response was attained on day- 11 of storage at $25^{\circ} \mathrm{C}$. Nevertheless, the electrochemical MIPs microspheres-based sensor response dropped to $40 \%$ of its initial response after 30 days of storage at ambient conditions. As such, the developed solid-state micro-MIPs-based SPE electrode possessed a life span of 11 days. 
RASĀYAN J. Chem.

Vol. 14 | No. 3 |1462-1468| July - September | 2021

\section{CONCLUSION}

The molecularly imprinted polymeric microspheres with high-affinity binding sites for cholesterol have been successfully synthesized with a simple emulsion photo-polymerization method. Detection of cholesterol with the electrochemical sensor based on micro-MIPs synthetic receptors provides a more economic procedure than enzymes or antibodies, which requires an expensive enzyme purification process and secretion of antibodies from animals. Thus, the robust biomimetic MIPs microsensor could serve as a feasible tool for selective and sensitive detection of cholesterol compared to conventional enzymatic biosensors and immunosensor that require immobilization of biological molecules.

\section{ACKNOWLEDGEMENT}

Thank you to the Universitas Negeri Padang for funding this research through the operational research grant with contract number 14411/UN35-13/LT/2020 and Universiti Pendidikan Sultan Idris Malaysia for instrument support at this research.

\section{REFERENCES}

1. H. Yang, L. Li, Y. Ding, D. Ye, Y. Wang, S. Cui and L. Liao, Biosensors and Bioelectronics, 95, 748 (2016), https://doi.org/10.1016/j.bios.2016.09.081

2. L.H. Li, E.P. Dutkiewicz, Y.C. Huang, H.B. Zhou and C.C. Hsu, Journal Food and Drug Analysis, xxx, 1(2018), https://doi.org/10.1016/j.jfda.2018.09.001

3. B. Sellergren and K.J. Shea, Journal of Chronramgmphy, 635, 31(1993), https://doi.org/10.1016/00219673(93)83112-6

4. O. Brüggemann, Advances in Biochemical Engineering/Biotechnology, 76, 127(2002), https://doi.org/10.1007/3-540-45345-8 4

5. B. Danielsson, Advances in Biochemical Engineering/Biotechnology, 109, 97(2008), https://doi.org/10.1007/10_2007_088

6. D. Futra, L.Y. Heng, M.Z. Jaapar, A. Ulianas, K. Saeedfara and T.L. Ling, Analytical Methods, 8, 1381(2016), https://doi.org/10.1039/c5ay02796a

7. A. Mulyasuryani and A. Savitri, Jurnal Kimia VALENSI: Jurnal Penelitian dan Pengembangan Ilmu Kimia, 1, 97(2015), https://doi.org/10.15408/jkv.v0i0.3146

8. J. Ji, Z. Zhou, X. Zhao, J. Sun, X. Sun, Biosensors and Bioelectronics, 66, 590(2014), https://doi.org/10.1016/j.bios.2014.12.014

9. N.A. Azis, I.M. Isa, N. Hashim, M.S. Ahmad, S. Nur, A.M. Yazid, M.I. Saidin, Suyanta, R. Zainul, A. Ulianas, S. Mukdasai, International Journal of Electrochemical Science, 14, 10607(2019), https://doi.org/10.20964/2019.11.46

10. M.S. Ahmad, I.M. Isa, N. Hashim, M.I. Saidin, Suyanta, R. Zainul, A. Ulianas, S. Mukdasai, International Journal of Electrochemical Science, 14, 9080(2019), https://doi.org/10.20964/2019.09.54

11. R.J. Bautista, W. Aperador, M.R. Joya, Rasayan Journal of Chemistry, 13, 2092(2020), https://doi.org/10.31788/RJC.2020.1345854

12. D.L. Pavia, G.M, Lampman, G.S. Kriz, J.A. Vyvyan, Introduction To Spectroscopy, Department of Chemistry Western Washington University Bellingham, Washington, p30, 4, (2009)

13. J. Wang, Analytical Electrochemistry, Las Cruces, New Mexico, 2, pp.121 (2000).

14. Deswati, S. Hamzar, Z. Rahmiana, P. Hilfi, Buchari, S. Henry, Rasayan Journal of Chemistry, 13, 2045(2020), https://doi.org/10.31788/RJC.2020.1345845

15. X. Ma and M. Chen, Sensors and Actuators B: Chemical, 215, 445(2015), https://doi.org/10.1016/j.snb.2015.04.016

16. N.A. Yusof, A. Beyan, M.J. Haron and N.A. Ibrahim, Sains Malaysiana, 39(5), 829 (2010).

17. Y. Zhai, Y. Liu, X. Chang, S. Chen, and X. Huang, Analytica Chimica Acta, 593, 123(2007), https://doi.org/10.1016/j.aca.2007.04.040

[RJC-6251/2020] 